\title{
Natural history of Danon disease
}

\author{
Dana Boucek, BA, Jean Jirikowic, MS, and Matthew Taylor, MD, PhD
}

\begin{abstract}
Background: Danon disease is a rare but serious cardiac and skeletal myopathy leading to substantial morbidity and early mortality due to arrhythmia and cardiomyopathy. The X-linked nature of inheritance accounts for reported differences in phenotypic severity between men and women. The rarity of Danon disease has limited understanding of the complete phenotype. Clinical estimates of ages of disease onset and survival based on gender have not been published. Methods and Results: We present data on 82 patients with Danon disease from 36 families, the largest series to date. Men were severely affected with cognitive disabilities (100\%), hypertrophic cardiomyopathy (88\%), and muscle weakness $(80 \%)$. Men had a high morbidity and were unlikely to reach the age of 25 years without a cardiac transplantation. Women were less severely affected but reported higher than expected levels of cognitive $(47 \%)$ and skeletal muscle complaints $(50 \%)$ and manifesting an equal prevalence of dilated cardiomyopathy and hypertrophic cardiomyopathy. Combining our data with that of 63 other Danon disease case reports in the literature, the average ages of first symptom, cardiac transplantation, and death were 12.1, 17.9, and 19.0 years in men and 27.9, 33.7, and 34.6 years in women, respectively. Conclusion: These data more broadly illuminate the Danon disease phenotype and should prove useful to physicians working with and providing genetic counseling to families with Danon disease. Women with Danon disease present with clinical symptoms and events approximately 15 years after men and report a higher proportion of cognitive and skeletal muscle problems than previously recognized. Genet Med 2011:13(6):563-568.
\end{abstract}

Key Words: cardiomyopathy, Danon disease, Wolff-Parkinson-White syndrome, genetics

$\mathrm{D}$ anon disease is a rare $\mathrm{X}$-linked dominant metabolic disorder initially described by Danon et al. ${ }^{1}$ in two unrelated 16-year-old boys manifesting cardiomyopathy, skeletal myopathy, and intellectual disability. In addition to this triad of findings, later reports showed that Danon disease led to retinal disease, hepatic disease, and pulmonary disease. ${ }^{2-6}$ The disease is now understood to be caused by a primary deficiency in lysosomeassociated membrane protein-2 (LAMP-2; OMIM\# 309060), ${ }^{7}$ which functions as a lysosomal membrane receptor in chaperonemediated autophagy. The precise pathophysiological mechanism through which LAMP-2 deficiency causes Danon disease is not well understood. It seems that all men and many women with LAMP2 gene mutations will develop cardiac disease including

From the Adult Medical Genetics Program, CU Cardiovascular Institute, University of Colorado Denver, Aurora, Colorado.

Matthew Taylor, MD, PhD, 12700 East 19th Avenue, Mailstop F442, Room 8022, Aurora, CO 80045. E-mail: matthew.taylor@ucdenver.edu.

Disclosure: The authors declare no conflict of interest.

Supplemental digital content is available for this article. Direct URL citations appear in the printed text and are provided in the HTML and PDF versions of this article on the journal's Web site (www.geneticsinmedicine.org).

Submitted for publication October 29, 2010

Accepted for publication December 9, 2010.

Published online ahead of print March 16, 2011

DOI: 10.1097/GIM.0b013e31820ad795 hypertrophic cardiomyopathy and dilated cardiomyopathy, cardiac preexcitation syndrome, and a propensity to arrhythmias. Clinical onset of disease in men occurs earlier, is a more aggressive disease course and men may succumb to sudden death or require cardiac transplantation in the second or third decade. A recent report of seven young patients, including one woman, highlighted the severity of Danon disease as it described either early death or heart transplant occurring in all six men before the age of 25 years; the sole female had reduced left-ventricular systolic function and required a cardiac defibrillator due to ventricular tachycardia at 22 years of age. ${ }^{8}$

Accounts of the Danon disease phenotype have been largely descriptive, stemming from case reports and modest-sized case series. An understanding of the phenotype in women with Danon disease is more limited compared with men. Data on the natural history of Danon disease including the average age of onset of disease and the survival course for men and women are lacking, rendering discussions of prognosis with patients and families challenging. ${ }^{69-12}$ Furthermore, the rarity of Danon disease ensures that physicians remain unfamiliar with the phenotype, and even large medical centers will evaluate at most a small numbers of patients. The largest and most detailed previous report of Danon disease described the cardiac and neuromuscular phenotype in 13 families. ${ }^{6}$

Our clinical genetics group began receiving inquiries from patients with Danon disease in 2002 and developed a Danon disease Registry effort in 2006. In this study, we report details of the clinical Danon disease phenotype and prognosis based on data from 82 individuals from 36 families with Danon disease that contacted our group. These data provide a broader overview of the Danon disease phenotype in men and women and permit estimates of survival to important clinical events that can be used by patients, physicians, and researchers to better recognize and understand Danon disease.

\section{METHODS}

Patients and families were identified through an adult medical genetics clinic, an ongoing cardiomyopathy research registry project, and through families and physicians contacting our adult medical genetics clinic and medical geneticist (M.T.) directly or by a patient-centered website about Danon disease (www.danondisease.org). The diagnosis of Danon disease was based on a combination of DNA analysis, immunohistochemistry, family history in each case, and medical records that were reviewed by our geneticist. In cases where a research DNA sample was provided, confirmatory LAMP2 DNA sequencing was done using standard Sanger sequencing of the nine LAMP2 exons as previously reported. ${ }^{11}$ As the number of individuals contacting our genetics clinic increased, a registry database for patients with Danon disease and their families was launched in 2006. The majority of the patients originated from the United States, Italy, Australia, Sweden, and the United Kingdom. Communication with subjects and their physicians was carried out primarily through e-mail and telephone correspondence or in person for those individuals who were able to travel to our site. Medical records on living and deceased individuals provided by subjects from each family were reviewed. 
A subset of patients who traveled to our center $(n=17)$ was enrolled in a research protocol that included medical history questionnaires $(n=27)$ and research examinations for those who traveled to our General Clinical Research Center. The in-person research evaluation included medical history, family history, physical examination, laboratory studies, electrocardiogram, echocardiogram, skin biopsy, and quantitative skeletal muscle testing. The medical history questionnaires asked questions about initial symptoms, method of diagnosis, cardiac studies performed, and organ- and system-based symptoms covering cardiovascular, muscle, cognitive (neurologic), ophthalmologic, gastrointestinal, pulmonary, and endocrine systems (Supplemental Digital Content 1, http://links.lww.com/GIM/A146). Data on specific events such as the age of symptom onset, age of diagnosis, and age at death or transplant were collected, and Kaplan-Meier log-rank survival curves for time to event were generated using PASW software (version 18; SPSS, Chicago, IL). The age of symptom onset was defined as the age when one of the following was first noted: cardiac or skeletal myopathy, cardiac preexcitation, or confirmed Danon disease. A PubMed search of English-language clinical reports on Danon disease where clinical information was presented was performed and used to provide a combined survival analysis of our data and that from the literature. Mutation data were used to ensure that the combined analysis did not duplicate data from those families in our study who had been previously in the literature. In families where mutation data were not available, the year of diagnosis, family structure, and medical center where the diagnosis was made were compared against published reports to avoid duplication of data in our cohort with published cases. Data are reported in aggregate to maintain confidentiality, informed consent was obtained from research subjects, and the Colorado Multiple Institutional Review Board approved the registry and research protocol.

\section{RESULTS}

Since 2002, more than 50 families contacted our group because of suspected Danon disease, and after our evaluation, 36 families, accounting for 82 patients, could be classified as having confirmed Danon disease. Diagnoses were confirmed when possible by review of the direct medical records, immunohistochemistry studies, clinical genetic testing reports, and/or DNA sequencing of $L A M P 2$ when a research DNA sample was available. We were contacted by patients in North and South America, Europe, and Australia. Given the wide dispersal of cases, much of our data collection occurred through telephone and e-mail sources; 17 subjects traveled to our site for in-person evaluations. Cases where the history from the patient and/or physician clearly indicated that confirmatory testing had been completed but the actual mutation report could not be provided by the patient or physician were included in our analysis. The availability of medical records and family history data were quite variable and incomplete in several cases, especially in the case of family history data for deceased family members. Cases where the clinical evidence and laboratory evidence were not confirmatory were labeled as "possible" or "unlikely" Danon disease and are not reported in this study. Cases where Danon disease was initially suspected but subsequent evaluation confirmed a different diagnoses included X-linked myopathy with excessive autophagy (one case), ultimately proven to be due to other diseases included one case each of X-linked excessive autophagy, hypertrophic cardiomyopathy due to a PRKAG2 mutation, and hydroxychloroquine induced myopathy.
Demographic, disease presentation, and outcome data are summarized in Table 1. As expected, men experienced events of symptom onset, diagnosis, and death or transplant earlier than women. The average age of onset and standard deviation of Danon disease symptoms was $12.1( \pm 6.5)$ years and $28.1( \pm 15)$ years in men and women, respectively. The average ages of diagnosis of disease, defined as skeletal or cardiac myopathy, cardiac preexcitation, or confirmed Danon disease (whichever occurred first), were $13.5( \pm 7.0)$ years and $31.4( \pm 15.4)$ years in men and women, respectively. Hypertrophic cardiomyopathy predominated in men, although some patients developed dilated disease. Previously, dilated cardiomyopathy was considered more prevalent in affected women; however, our data revealed nearly equal rates of dilated cardiomyopathy versus hypertrophic cardiomyopathy in women. ${ }^{6}$ Conduction abnormalities were reported in more than three quarters of men and women; $68 \%$ of men had a Wolff-Parkinson-White pattern on electrocardiography in comparison with $27 \%$ of women with a WolffParkinson-White pattern. Confirmed genetic results were available in 18 families (Table 2), with the other families having their diagnoses confirmed on the basis of LAMP-2 immunostaining, available medical records, and/or direct communications with treating physicians. The majority of mutations are predicted to cause LAMP-2 deficiency (frameshifts, stop codons, and splicing site mutations). Most mutations are private, being reported in just a single family with exceptions notable for recurrent mutations in unrelated families in exons 3 (Q83X, W98X) and 7 (R293X, V310I [frameshift]).

Clinical symptom data abstracted from available medical records and histories sufficiently complete for analysis are summarized in Table 3. Learning or cognitive disorders were reported in $100 \%$ of men and, surprisingly, in approximately $50 \%$ of women. Cognitive disabilities were mostly mild to moderate in men, with severe "mental retardation" being infrequent; in women, mostly mild and nonspecific cognitive complaints were noted. Symptomatic heart disease was the most common symptom in women and the second most frequent symptom in men (behind cognitive complaints); however, symptomatic heart disease was reported more often in men, when compared with

Table 1 Danon disease population

\begin{tabular}{lcr}
\hline & $\begin{array}{c}\text { Men } \\
(n=43)^{a}\end{array}$ & $\begin{array}{c}\text { Women } \\
(n=39)\end{array}$ \\
\hline Symptomatic clinical disease & $37(94.9)$ & $31(91.2)$ \\
Clinical diagnosis ${ }^{b}$ & $39(100)$ & $32(94.1)$ \\
Cardiac transplant & $13(33.3)$ & $6(17.6)$ \\
Living & $23(59.0)$ & $23(67.6)$ \\
Mean age (yr) & & \\
$\quad$ First symptom & $11.7( \pm 6.4)$ & $26.8( \pm 14.2)$ \\
$\quad$ Diagnosis & \\
$\quad$ Cardiac transplant & $13.1( \pm 7.0)$ & $30.9( \pm 15.2)$ \\
$\quad$ Death & $20.8( \pm 6.7)$ & $32.3( \pm 14.5)$ \\
\hline
\end{tabular}

${ }^{a}$ Subject numbers reflect subjects where complete data were available (percentages or standard deviations are given in parentheses).

${ }^{b}$ Diagnosis of cardiac or skeletal myopathy, cardiac preexcitation, or confirmed Danon disease (whichever came first). In two male and one female cases, cardiac abnormalities were detected by screening before the onset of symptomatic clinical disease. 
Table 2 Reported mutations

\begin{tabular}{|c|c|c|c|}
\hline Location & Mutation & Effect/mechanism & Reference \\
\hline Promoter & c.-22_30delCGCCGCCGT & & This study \\
\hline Exon 1 & c.36_42delAGGGCTC & Frameshift & 13 \\
\hline Exon 1 & c.1-?_64+? & Large deletion & This study \\
\hline IVS-1 & c. $64+1 \mathrm{G}>\mathrm{A}$ & Splicing & This study \\
\hline IVS-1 & c. $64+1 \mathrm{G}>\mathrm{T}$ & Splicing & 8 \\
\hline Exon 2 & c.102-103 delAG & Frameshift & 9 \\
\hline Exon 2 & c. $138 \mathrm{G}>\mathrm{A}$ & Stop codon & 14 \\
\hline Exon 2 & c. 179 delC & Frameshift & 15 \\
\hline IVS-2 & c. $183+1 \mathrm{G}>\mathrm{A}$ & Splicing & This study \\
\hline Exon 3 & c. $247 \mathrm{C}>\mathrm{T}$ & Stop codon & This study (2 families) \\
\hline Exon 3 & c. $294 \mathrm{G}>\mathrm{A}$ & Stop codon & This study ( 2 families) and 16 \\
\hline Exon 3 & c. $327 \mathrm{~T}>\mathrm{A}$ & Stop codon & 8 \\
\hline Exons 4-10 & c.398-?_1233+?del & Large deletion & This study \\
\hline Exon 4 & c. $467 \mathrm{~T}>\mathrm{G}$ & Stop codon & 12 \\
\hline Exon 4 & c. $470 \mathrm{C}>\mathrm{G}$ & Stop codon & 10 \\
\hline Exon 4 & c. $507 \mathrm{G}>\mathrm{A}$ & Stop codon & This study \\
\hline Exon 4 & c. $520 \mathrm{C}>\mathrm{T}$ & Stop codon & 13 \\
\hline Exon 5 & c.573 delA & Frameshift & 17 \\
\hline Exon 5 & c.716 delT & Frameshift & 18 \\
\hline IVS-5 & c.742-4_742+6 delGAAGGTTGCT & Splicing & 19 \\
\hline IVS-6 & c. $864+1-4$ del GTGA & Splicing & 8 \\
\hline IVS-6 & c. $865-1 \mathrm{G}>\mathrm{C}$ & Splicing & This study \\
\hline IVS-6 & c. $865-2 \quad A>G$ & Frameshift & 8 \\
\hline IVS-6 & c. $865-3 \mathrm{C}>\mathrm{A}$ & Splicing & 20 \\
\hline Exon 7 & c.874-897del AACCGATTTTATCTGAAGGAAGTG & Large deletion & 3 \\
\hline Exon 7 & c. $877 \mathrm{C}>\mathrm{T}$ & Stop codon & This study (2 families) \\
\hline Exon 7 & c. $883-884$ ins T & Frameshift & 21 \\
\hline Exon 7 & c. $928 \mathrm{G}>\mathrm{A}$ & Splicing & This study, $8,18,22$, and 23 \\
\hline Exon 8 & c. $961 \mathrm{~T}>\mathrm{C}$ & Nonsynonymous & 24 \\
\hline Exon 8 & c. $1075 \mathrm{C}>\mathrm{T}$ & Stop codon & 12 \\
\hline Exon 8 & c. 1082 delA & Frameshift & This study \\
\hline IVS-8 & c. $1093+1 \mathrm{G}>\mathrm{C}$ & Splicing & 25 \\
\hline Exon 9B & c.-1137-1140 del TATA/ins GCTGGTCCCAAT & Insertion/deletion & This study \\
\hline
\end{tabular}

women ( $89 \%$ vs. $8 \%$ ). Palpitations were the most universally reported cardiovascular complaint followed by chest pain. Cardiac ablation procedures were performed on one half of men and one third of women. Implantable defibrillators were placed in $41 \%$ and $31 \%$ of men and women, respectively; data on rates of appropriate discharge could not be ascertained. Symptomatic muscle disease was reported by more men than women, whereas neuropathy was reported three times more often in women than men.
Symptomatic respiratory disease was more frequent in men than women, with asthma being reported most often. Restrictive lung problems, possibly related to respiratory muscle weakness, were noted in just two male patients. Gastrointestinal complaints were more common in men and included chronic abdominal pain, constipation, and diarrhea. Esophageal dysmotility was reported by one woman. Laboratory abnormalities present in many male patients included elevations of creatine kinase (1.8- to 7.4-fold over upper limits of normal), alanine 
Table 3 Reported clinical characteristics

\begin{tabular}{|c|c|c|}
\hline & $\begin{array}{c}\text { Men } \\
(n=26)\end{array}$ & $\begin{array}{l}\text { Women } \\
(n=18)\end{array}$ \\
\hline \multicolumn{3}{|l|}{ Cardiac } \\
\hline Symptomatic heart disease & 88.5 & 77.7 \\
\hline Chest pain & 41.6 & 37.5 \\
\hline Palpitations & 76.5 & 68.8 \\
\hline $\begin{array}{l}\text { Hypertrophic } \\
\text { cardiomyopathy }\end{array}$ & 88 & 33.3 \\
\hline Dilated cardiomyopathy & 12 & 27.7 \\
\hline Conduction abnormality & 86.4 & 80 \\
\hline Wolf-Parkinson-White & 68.2 & 26.7 \\
\hline Cardiac ablation & 53.3 & 30.8 \\
\hline Defibrillator implantation & 41.2 & 31.3 \\
\hline \multicolumn{3}{|l|}{ Neurologic } \\
\hline $\begin{array}{l}\text { Learning and cognitive } \\
\text { problems }\end{array}$ & 100 & 46.6 \\
\hline $\begin{array}{l}\text { Visual and retinal } \\
\text { abnormalities }\end{array}$ & 69.2 & 64.2 \\
\hline $\begin{array}{l}\text { Symptomatic muscle } \\
\text { disease }\end{array}$ & 80 & 50 \\
\hline Muscle cramping & 9.1 & 15.3 \\
\hline Neuropathy & 9.1 & 38.5 \\
\hline \multicolumn{3}{|l|}{ Respiratory } \\
\hline $\begin{array}{l}\text { Symptomatic respiratory } \\
\text { disease }\end{array}$ & 50 & 16.7 \\
\hline \multicolumn{3}{|l|}{ Gastrointestinal } \\
\hline Symptomatic GI disease & 76.5 & 50 \\
\hline \multicolumn{3}{|l|}{ Other } \\
\hline Hypertension & 9.1 & 17.6 \\
\hline Hyperlipidemia & 20 & 35.7 \\
\hline \multicolumn{3}{|l|}{ Laboratory values } \\
\hline Creatine kinase $(\mathrm{U} / \mathrm{L})$ & $943.7( \pm 326.8)$ & $105.6( \pm 104.4)$ \\
\hline $\begin{array}{l}\text { Asparate aminotransferase } \\
\text { (U/L) }\end{array}$ & $290.4( \pm 108.7)$ & $55.8( \pm 41.8)$ \\
\hline $\begin{array}{l}\text { Alanine aminotransferase } \\
(\mathrm{U} / \mathrm{L})\end{array}$ & $234( \pm 101.6)$ & $37( \pm 20.9)$ \\
\hline
\end{tabular}

The symptomatic data are presented as a percentage of affected individuals for whom data were available, and the laboratory values are presented as a mean ( \pm standard deviation).

aminotransferase (2.4- to 10.4-fold), and aspartate aminotransferase (3.9- to 12.4-fold). In women, the average aminotransferase levels were normal to slightly elevated ( $<2$-fold). Creatine kinase values were normal in women with the exception of one woman who had a 2-fold elevation.

In each confirmed case, we asked specifically about age of symptom onset, diagnosis of cardiac and/or skeletal myopathy, transplantation, and death. Complete data from living patients and data that could be confirmed from medical records of deceased individuals were used to estimate survival curves for men and women (Fig. 1). The survival analysis showed that men fared worse than women overall in terms of their age of first symptom, diagnosis of cardiac or skeletal myopathy, cardiac transplantation, and death. In our cohort, the mean ages in years and standard deviations of cardiac transplantation, and death were $18.1( \pm 5.9)$ and $19.8( \pm 7.1)$ in men and 34.5 $( \pm 15.7)$ and $36.0( \pm 16.0)$ in women, respectively.

A PubMed search for reports on Danon disease where sufficient clinical data on these same parameters were provided yielded 63 additional cases (40 men and 23 women) from 20 published studies (Table 2). For the combined data from our study and the 63 cases from the literature (Fig. 1), the mean ages in years ( \pm standard deviation) of the first symptom, diagnosis of cardiac or skeletal myopathy, cardiac transplantation, and death were $12.1( \pm 6.8), 12.8( \pm 7.0), 17.9( \pm 7.2)$, and 19.0 $( \pm 8.0)$ in men and $27.9( \pm 14.5), 29.9( \pm 14.7), 33.7( \pm 16.1)$, and $34.6( \pm 15.5)$ in women, respectively. All together, $59.0 \%$ of men and $51.6 \%$ of women reached an endpoint of transplant or death. The oldest living male who did not require a transplant or die was reported to be of age 41 years. ${ }^{20}$ His mutation was a splicing mutation that is less typical of Danon disease and could have potentially led to some residual protein function; however, the authors were unable to demonstrate any LAMP-2 protein on Western blot analysis. If this atypical case is excluded, then the next oldest male in our study who did not reach a transplant or death endpoint was 25 years, highlighting the life-threatening nature of Danon disease in men by the third decade.

\section{DISCUSSION}

The rarity of Danon disease and its unfamiliarity of physicians have limited progress in understanding the phenotype and natural history of this severe disease. The exact prevalence is unknown, although $L A M P 2$ mutations were noted in $1 \%$ of cases of hypertrophic cardiomyopathy. ${ }^{13}$ Given the low prevalence, a minority of physicians would be expected to be familiar with the diagnosis, and even at specialized centers, the total number of patients seen is small. Sugie et al. ${ }^{6}$ authored the best previously published phenotype description, combining data from six families with seven previously reported in the literature. The other notable "large" case series recently described the extensive and severe cardiac phenotype in one female and six male cases. ${ }^{8}$ Our results on 82 patients from 36 families (the largest series to date) demonstrate a high prevalence of cardiac disease in both men (88.5\%) and women (77.7\%) with a delay in the onset and diagnosis of disease in women by 15-20 years, when compared with men. These data extend prior work to a larger population size and present endpoint analysis data that paint a picture of the progression and prognosis of Danon disease in men and women.

Danon disease is clearly a serious health problem for affected men where survival beyond 25 years without a cardiac transplant is improbable. Young males with progressive cardiomyopathy should, therefore, be evaluated promptly for the inevitability of cardiac transplantation. Notably, our data show that women also are significantly affected with symptomatic heart disease and conduction disease and seem at equal risk for either dilated cardiomyopathy or hypertrophic cardiomyopathy. The prevalence of dilated cardiomyopathy and hypertrophic cardiomyopathy in women was essentially equivalent in our study, a finding somewhat in contrast to the previous estimate where dilated cardiomyopathy was thought to be the dominant $(70 \%)$ cardiac phenotype for women. ${ }^{6}$ However, as reports of evolu- 


\section{Study $\quad$ First Symptom $\quad$ Combined}
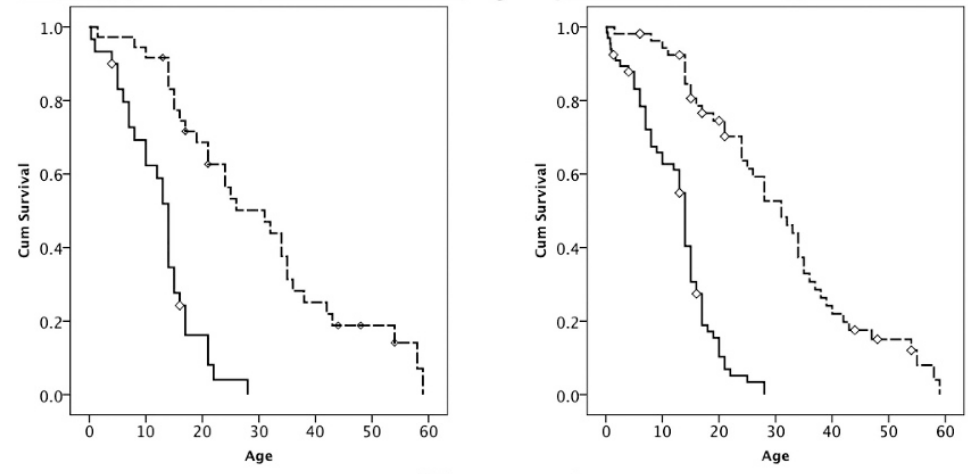

\section{Diagnosis}
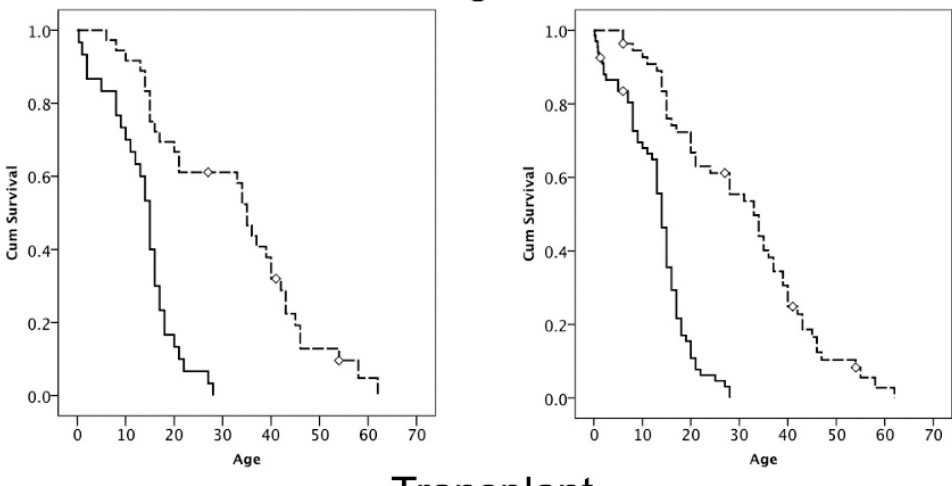

Transplant
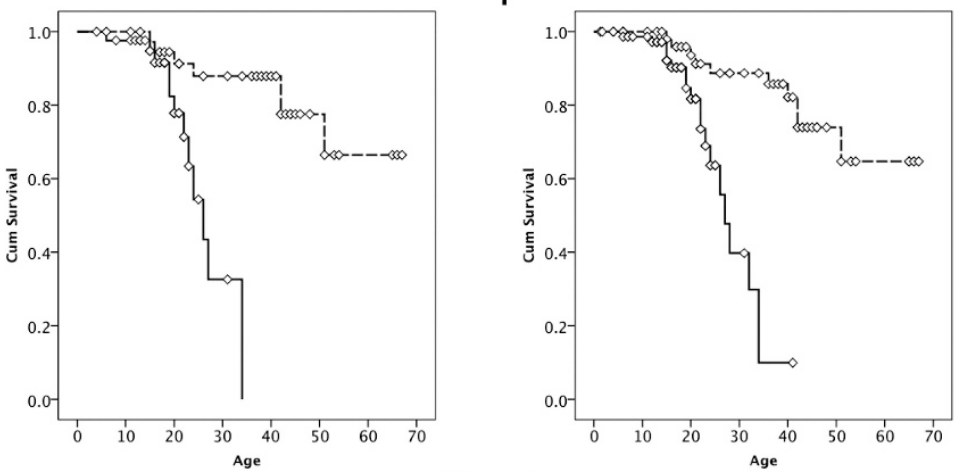

Death
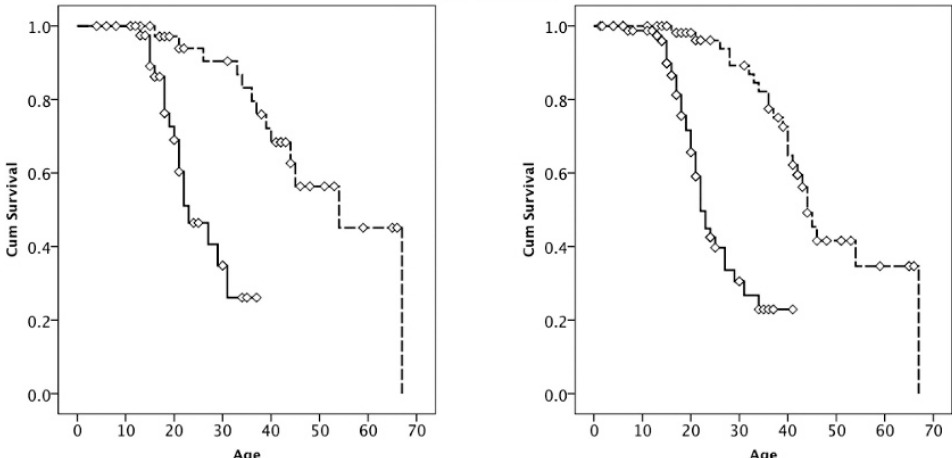

Fig. 1. Kaplan-Meier survival curves for the endpoints in years of first symptom, diagnosis of cardiac and/or skeletal myopathy, cardiac transplantation, and death. The left column depicts data from this study, and the right column shows combined data from our study and 63 additional cases from the literature. References for literature studies where mutations were reported are listed in Table 2. Curves for men and women are depicted by hashed and solid lines, respectively. Censored time points are indicated with diamonds. All pair-wise comparisons between men and women were significant $(P<0.001)$ by the log-rank test. 
tion of hypertrophic phenotypes to more dilated presentations have been described in women, it is possible that the equal ratio of hypertrophic to dilated cases in our cohort may evolve over time. Additional clinical features not previously reported by others include the high rates of learning problems, visual problems, muscle disease, neuropathy, and gastrointestinal disease in women. Our survival analysis provides the first estimates of the occurrence of important clinical endpoints and further delineates the significant morbidity and mortality risks that apply to women with Danon disease. These data can be used by physicians to counsel and educate patients about the prognosis and treatment of Danon disease.

Importantly, our registry study is limited by the problems common to studying rare diseases. Most significantly, the majority of data were obtained through medical history and provided medical records as patients contacting us were widely dispersed across four continents, North and South America, Europe, and Australia. Understandably, data ascertainment was neither uniform nor systematic given the different information available in each case. Geographic constraints meant that in the majority of cases, we relied on direct communication with patients and physicians and provided medical records, which were often incomplete. Travel funds to fly subjects to our site were not available; thus, only 17 subjects were able to travel to our site for direct in-person evaluations. In most cases, echocardiogram and the few cardiac magnetic resonance imaging files were unavailable for our review and are not reported in this study. Understandably, the majority of our data comes from English-speaking patients, and we cannot exclude the possibility that the prevalence of Danon disease differs in other geographic and ethnic groups. Our data represent a step to understand and characterize the phenotype of this rare condition whose prevalence is as yet unknown. We suspect that given the unfamiliarity of Danon disease to the majority of health care providers, a substantial number of patients remain undiagnosed.

Our survival estimates are based on living patients and family history data, which included deceased relatives, some of whom died before a formal diagnosis of Danon disease was reached. Enhancements in cardiac treatment, transplant, and defibrillator therapy have likely improved overall outcomes in Danon disease, which could mean that survival chances today may be more favorable than our estimates herein, which included data from distantly deceased family members. The ability to diagnose Danon disease with modern molecular methods along with reports of the severity of the phenotype herein and by others should further encourage physicians to consider definitive transplant therapy in confirmed cases. ${ }^{8}$ As molecular genetic testing panels for unexplained cardiomyopathy are increasingly used, the number of known Danon cases will expand permitting future studies to address how the various medical and surgical interventions alter the natural history of Danon disease in men and women.

\section{ACKNOWLEDGMENTS}

This work was supported by the Muscular Dystrophy Association (MDA67944). The authors express their gratitude to the patients and families with Danon disease.

\section{REFERENCES}

1. Danon MJ, Oh SJ, DiMauro S, et al. Lysosomal glycogen storage disease with normal acid maltase. Neurology 1981;31:51-57.

2. Fanin M, Nascimbeni AC, Fulizio L, Spinazzi M, Melacini P, Angelini C. Generalized lysosome-associated membrane protein-2 defect explains multisystem clinical involvement and allows leukocyte diagnostic screening in Danon disease. Am J Pathol 2006;168:1309-1320.

3. Lacoste-Collin L, Garcia V, Uro-Coste E, et al. Danon's disease (X-linked vacuolar cardiomyopathy and myopathy): a case with a novel Lamp-2 gene mutation. Neuromuscul Disord 2002;12:882-885.

4. Prall FR, Drack A, Taylor M, et al. Ophthalmic manifestations of Danon disease. Ophthalmology 2006;113:1010-1013.

5. Schorderet DF, Cottet S, Lobrinus JA, Borruat FX, Balmer A, Munier FL. Retinopathy in Danon disease. Arch Ophthalmol 2007;125:231-236.

6. Sugie K, Yamamoto A, Murayama K, et al. Clinicopathological features of genetically confirmed Danon disease. Neurology 2002;58:1773-1778.

7. Nishino I, Fu J, Tanji K, et al. Primary LAMP-2 deficiency causes X-linked vacuolar cardiomyopathy and myopathy (Danon disease). Nature 2000;406: 906-910.

8. Maron BJ, Roberts WC, Arad M, et al. Clinical outcome and phenotypic expression in LAMP2 cardiomyopathy. JAMA 2009;301:1253-1259.

9. Echaniz-Laguna A, Mohr M, Epailly E, et al. Novel Lamp-2 gene mutation and successful treatment with heart transplantation in a large family with Danon disease. Muscle Nerve 2006;33:393-397.

10. Lobrinus JA, Schorderet DF, Payot M, et al. Morphological, clinical and genetic aspects in a family with a novel LAMP-2 gene mutation (Danon disease). Neuromuscul Disord 2005;15:293-298.

11. Taylor MR, Ku L, Slavov D, et al. Danon disease presenting with dilated cardiomyopathy and a complex phenotype. J Hum Genet 2007:52:830-835.

12. Yang Z, McMahon CJ, Smith LR, et al. Danon disease as an underrecognized cause of hypertrophic cardiomyopathy in children. Circulation 2005; 112:1612-1617.

13. Charron P, Villard E, Sebillon P, et al. Danon's disease as a cause of hypertrophic cardiomyopathy: a systematic survey. Heart 2004;90:842-846.

14. Balmer C, Ballhausen D, Bosshard NU, et al. Familial X-linked cardiomyopathy (Danon disease): diagnostic confirmation by mutation analysis of the LAMP2gene. Eur J Pediatr 2005;164:509-514.

15. Regelsberger G, Hoftberger R, Pickl WF, et al. Danon disease: case report and detection of new mutation [published online ahead of print July 7, 2009]. $J$ Inherit Metab Dis doi: 10.1007/s10545-009-1097-9.

16. Fanin M, Nascimbeni AC, Fulizio L, Spinazzi M, Melacini P, Angelini C. Generalized lysosome-associated membrane protein-2 defect explains multisystem clinical involvement and allows leukocyte diagnostic screening in Danon disease. Am J Pathol 2006;168:1309-1320.

17. Dougu N, Joho S, Shan L, et al. Novel LAMP-2 mutation in a family with Danon disease presenting with hypertrophic cardiomyopathy. Circ $J 2009$; 73:376-380

18. Sabourdy F, Michelakakis H, Anastasakis A, et al. Danon disease: further clinical and molecular heterogeneity. Muscle Nerve 2009;39:837-844.

19. Sugie K, Koori T, Yamamoto A, et al. Characterization of Danon disease in a male patient and his affected mother. Neuromuscul Disord 2003;13:708711

20. Nadeau A, Therrien C, Karpati G, Sinnreich M. Danon disease due to a novel splice mutation in the LAMP2 gene. Muscle Nerve 2008;37:338-342.

21. Takahashi M, Yamamoto A, Takano K, et al. Germline mosaicism of a novel mutation in lysosome-associated membrane protein-2 deficiency (Danon disease). Ann Neurol 2002;52:122-125.

22. Bertini E, Donati MA, Broda P, et al. Phenotypic heterogeneity in two unrelated Danon patients associated with the same LAMP-2 gene mutation. Neuropediatrics 2005;36:309-313.

23. Burusnukul P, de Los Reyes EC, Yinger J, Boue DR. Danon disease: an unusual presentation of autism. Pediatr Neurol 2008;39:52-54.

24. Musumeci O, Rodolico C, Nishino I, et al. Asymptomatic hyperCKemia in a case of Danon disease due to a missense mutation in Lamp-2 gene. Neuromuscul Disord 2005;15:409-411.

25. Di Blasi C, Jarre L, Blasevich F, Dassi P, Mora M. Danon disease: a novel LAMP2 mutation affecting the pre-mRNA splicing and causing aberrant transcripts and partial protein expression. Neuromuscul Disord 2008;18: $962-966$. 